\title{
Diastereoselective Synthesis of Diamino 1,2-Diols from Homochiral $\alpha$-Aminoacylsilanes
}

Bianca F. Bonini, Mauro Comes-Franchini, Mariafrancesca Fochi, Lodovico Lunazzi, Andrea Mazzanti, Germana Mazzanti, Alfredo Ricci,* Greta Varchi

Dipartimento di Chimica Organica "A. Mangini", Università di Bologna, Viale Risorgimento 4, 40136-Bologna, Italy

Fax +39 51 2093654; E-mail: ricci@ms.fci.unibo.it

Received 01 January 2001

Abstract: We have developed a new synthetic access to stereodefined diamino 1,2-diols starting from homochiral $\alpha$-aminoacylsilanes. A [3+2] cycloaddition with benzo nitrile oxide of the vinylated adducts and a reductive ring opening constitute key steps of the reaction sequence.

Key words: $\alpha$-aminoacylsilanes, vinylation, dipolar cycloaddition, aminopolyols

Stereodefined hydroxy amino alcohols are well recognised as key components for a variety of protease inhibitors and other new generation pharmaceutics and as ligands for asymmetric catalysis. ${ }^{1}$ We recently reported ${ }^{2}$ the stereoselective synthesis of functionalized amino alcohol units and of statine analogs via three- and two-carbon elongations respectively, from $\alpha$-aminoacylsilanes. The use of these synthetic equivalents of aldehydes, allows to overcome $^{2}$ the serious problems due to the easy racemization and avoids the special precautions necessary for the synthesis, handling and storing frequently encountered with $\alpha$-amino aldehydes. ${ }^{3}$ In this paper we present a new approach to the two-carbon homologation of acylsilanes based on the delivery of a vinyl unit to a carbonyl group. The reaction under study is aimed at the synthesis of stereodefined aminopolyols possessing the core-unit present in dihydroxypropylamine derivatives capable of highly efficient renin ${ }^{4}$ and HIV-1 protease ${ }^{5}$ inhibitions (Fig.1).

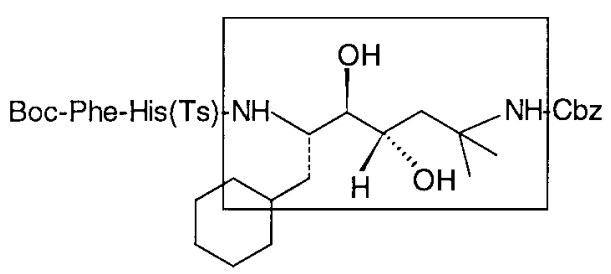

Figure 1

Our approach started with the homochiral aminoacylsilanes $\mathbf{1}$ and $\mathbf{2}$ derived from phenylalanine and isoleucine. ${ }^{2}$ Since the addition of vinylmagnesium and lithium organometallics to acylsilanes studied by Kuwajima ${ }^{6}$ gives rise to extensive amounts of silyl enol ethers along with the expected allylic alcohols, in a previous paper ${ }^{7}$ we devised a convenient new entry to silylated allylic alcohols from acylsilanes by using the magnesium to cerium transmetallation technique.

Treatment of $\mathbf{1}$ and $\mathbf{2}$ with the vinylmagnesium bromide/ $\mathrm{CeCl}_{3}$ complex, led (Scheme 1) to the formation of adducts $\mathbf{3}$ and $\mathbf{4}$ in good isolated yields and high diastereoselectivities $(99 \%$ d.e. $){ }^{8}$<smiles>[R]C(NC(=O)OCc1ccccc1)C(=O)O[R16](=O)c1ccccc1</smiles>

$$
\begin{array}{ll}
1: \mathrm{R}=\mathrm{PhCH}_{2}, & 3: 80 \%, 99 \% \text { d.e. } \\
2: \mathrm{R}= & 4: 75 \%, 99 \% \text { d.e. }
\end{array}
$$

Scheme 1

For introducing new hydroxy and amino functionalities in the vinyl moiety we envisioned the $[3+2]$ cycloaddition with nitriloxides. Cycloadditions of this type are usually regarded as mildly electrophilic in character. Therefore ( $\alpha$-hydroxyallyl)silanes in which the preferred location of allylic substituents maximizes ${ }^{9}$ electron donation should act, as shown by Curran, ${ }^{10}$ as efficient dipolarophiles. When 3 and $\mathbf{4}$ were reacted with the in situ prepared benzo nitrile oxide, isoxazolines $\mathbf{5}$ and $\mathbf{6}$ were obtained in $70 \%$ and $55 \%$ yields. In contrast with the modest diastereomeric excess $(20 \%)$ observed in the case of $\mathbf{5}$, only one diastereoisomer ( $99 \%$ d.e.) could be detected and isolated in the case of $\mathbf{6}$. The diastereomeric couple $\mathbf{5 a}, \mathbf{b}$ could be

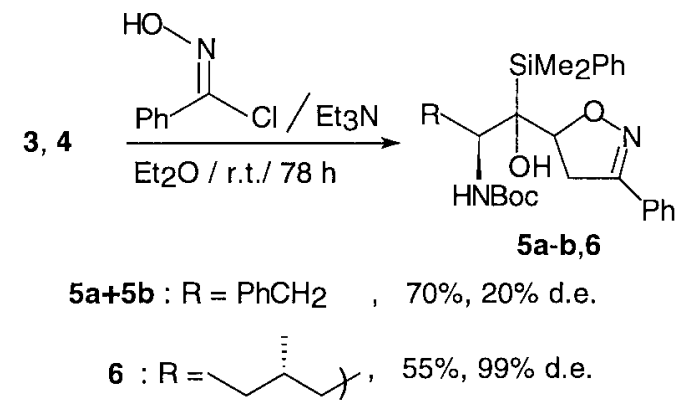

Scheme 2 
separated by column chromatography on silica gel and characterized. ${ }^{11}$

The subsequent desilylation of the $\alpha$-hydroxysilane cycloadducts carried out on a model compound, was disappointing. Under the action of bases the $\beta$-elimination of silanol led to ring opening with generation of an enolic form which upon rearrangement resulted in the formation of the desilylated hydroxylamino derivative 7 (Scheme 3).

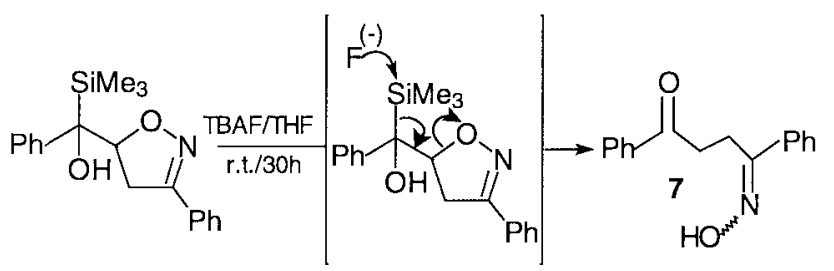

Scheme 3

To prevent the occurrence of the above mentioned Peterson elimination reaction from the $\beta$-hydroxysilane moiety ${ }^{12}$ in the presence of strongly basic reagents, isoxazolines 5a, the major diastereoisomer, and $\mathbf{6}$ were subjected to an oxidative desilylation. This reaction, although already reported in the literature,,$^{13}$ has been however scarcely investigated. Since the oxidation with pyridinium dichromate (PDC) has found an useful and high yielding application in the synthesis of acylsilanes from 1,1-disilyl alcohols, ${ }^{13 b}$ we applied this procedure to the desilylation of 5a and 6. Upon treatment with PDC at room temperature, these compounds underwent (Scheme 4) smooth desilylative oxidation to give $\mathbf{8}$ and $\mathbf{9}$ in high yields and virtually diastereomerically pure. ${ }^{14}$

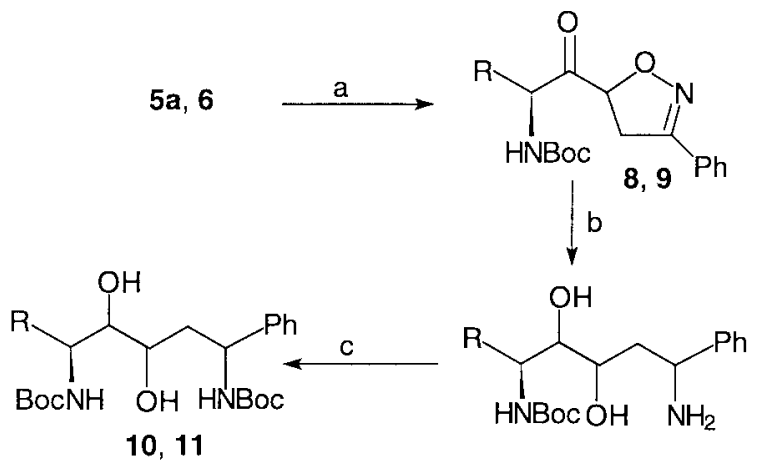

5a, 8, 10: $\mathrm{R}=\mathrm{CH}_{2}-\mathrm{Ph}$

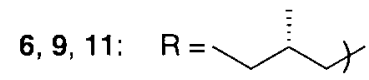

Scheme 4 a) PDC, DMF, r.t., 18 h, $98 \%$ yield, $99 \%$ d.e. for both 8 and 9; b) $\mathrm{NaBH}_{4}, \mathrm{NiCl}_{2} \bullet 6 \mathrm{H}_{2} \mathrm{O}, \mathrm{MeOH},-30{ }^{\circ} \mathrm{C}, 65 \mathrm{~h}$; c) $\mathrm{Boc}_{2} \mathrm{O}, \mathrm{Me}-$ $\mathrm{OH}, \mathrm{Et}_{3} \mathrm{~N}$, r.t., 4 days, $35 \%$ for $\mathbf{1 0}$ and $30 \%$ for $\mathbf{1 1}$.

The reductive ring opening of the oxazolidine ring aimed at the formation of the new hydroxy and amino functionalities was then performed. To avoid reaction conditions able to promote an acidic proton abstraction, we exploited the use of $\mathrm{NaBH}_{4}$ in methanol in the presence of $\mathrm{NiCl}_{2} \cdot 6 \mathrm{H}_{2} \mathrm{O}$. Exposure of 8 and 9 to this reagent at $-30{ }^{\circ} \mathrm{C}$ according to the literature ${ }^{15}$ led to heterocyclic ring opening and reduction of the carbonyl function and afforded after $65 \mathrm{~h}$, the expected aminopolyols. These were directely converted to the corresponding bis-Boc derivatives: in both cases one diastereoismer was found to largely prevail which was isolated by column chromatography and characterized. ${ }^{16}$ By this procedure N-Boc protected aminopolyols $\mathbf{1 0}$ and $\mathbf{1 1}$ are produced in 35\% and 30\% overall yields in three steps from oxazolidines $\mathbf{5 a}$ and $\mathbf{6}$ respectively. Connectivity of diamino 1,2-diols $\mathbf{1 0}$ and $\mathbf{1 1}$ was achieved by homonuclear and heteronuclear correlation. The stereochemical assignment of the three unknown stereogenic centers of $\mathbf{1 1}$ was attempted on the basis of 2DNOESY constraints and molecular modelling. ${ }^{17}$ The 15 observed constraints restricted the choice to two possible solutions: in both cases the three unknown centres are expected to display the same chirality and either configuration SSSSS or SSRRR, could be considered for 11 (Figure 2). To solve this ambiguity a single crystal X-ray diffraction ${ }^{18}$ was performed. The configuration was found to be SSSSS (Figure 2, left). Work is in progress to establish also the stereochemistry of compound $\mathbf{1 0 .}$.

In conclusion we have established the viability of a new short route to diamino 1,2-diols starting from homochiral $\alpha$-aminoacylsilanes. The importance of the target compounds highlights the significance of this new synthetic protocol in which in a single reductive-unfolding step, three new functional groups are generated.

\section{Acknowledgement}

This work has been supported by University of Bologna (Funds for Selected Research Topics, A.A. 1999-2001) and by the National Project "Stereoselezione in Sintesi Organica. Metodologie ed Applicazioni 1999-2001".

\section{References and Notes}

(1) Pastò, M.; Moyano, A.; Pericàs, M.A.; Riera, A. Tetrahedron: Asymmetry 1996, 7, 243, and references therein.

(2) (a) Bonini, B.F.; Comes-Franchini, M.; Fochi, M.; Gawronsky, J.; Mazzanti, G.; Ricci, A.; Varchi, G. Eur. J. Org. Chem. 1999, 437; (b) Bonini, B.F.; Comes-Franchini, M.; Fochi, M.; Laboroi, F.; Mazzanti G.; Ricci, A.; Varchi, G. J. Org. Chem. 1999, 64, 8008.

(3) (a) Coy, D.H.; Hocart, S.J. Tetrahedron 1988, 44, 835; (b) Jurczak, J.; Golebiowski Chem. Rev. 1989, 89, 149.

(4) Luly, J.R.; Maung, N.B.; Soderquist, J.; Fung, A.K.L.; Stein, H.; Kelinert, H.D.; Marcotte, P.A.; Egan, D.A.; Bopp, B.; Meirts, I.; Bolis, G.; Greer, J.; Perun, T.J.; Plattner, J. J. Med. Chem. 1988, 31, 2264.

(5) Thaisrivongs, S. Int. Pat. Appl. WO 91/01327, 1991.

(6) Kato, M.; Mori, A.; Oshino, H.; Ende, J.; Kobayashi, K.; Kuwajima, I. J. Am. Chem. Soc. 1984, 106, 1773.

(7) Bonini, B.F.; Comes-Franchini, M.; Fochi, M.; Mazzanti, G.; Ricci, A.; Varchi, G. Synlett 2000, 1688.

(8) 3: Yellowish oil: $[\alpha]_{\mathrm{D}}{ }^{20}=-49.3\left(c=2.0, \mathrm{CHCl}_{3}\right) ;{ }^{1} \mathrm{H} \mathrm{NMR}$ $\left(200 \mathrm{MHz}, \mathrm{CDCl}_{3}\right) \delta: 0.38\left(\mathrm{~s}, 3 \mathrm{H}, \mathrm{Si}-\mathrm{CH}_{3}\right), 0.40(\mathrm{~s}, 3 \mathrm{H}, \mathrm{Si}-$ $\left.\mathrm{CH}_{3}\right), 1.24\left(\mathrm{~s}, 9 \mathrm{H}, \mathrm{C}\left(\mathrm{CH}_{3}\right)_{3}\right), 1.46(\mathrm{~s}, 1 \mathrm{H}, \mathrm{OH}), 2.54(\mathrm{dd}, 1 \mathrm{H}$, $\left.J=13.6,10.4 \mathrm{~Hz}, \mathrm{CH}_{2}-\mathrm{Ph}\right), 2.90(\mathrm{dd}, 1 \mathrm{H}, J=13.6,3.6 \mathrm{~Hz}$, 
<smiles>CC[C@H](C)[C@H](NC(=O)OC(C)(C)C)[C@@H](O)[C@@H](O)C[C@@H](NC(=O)OC(C)(C)C)c1ccccc1</smiles>

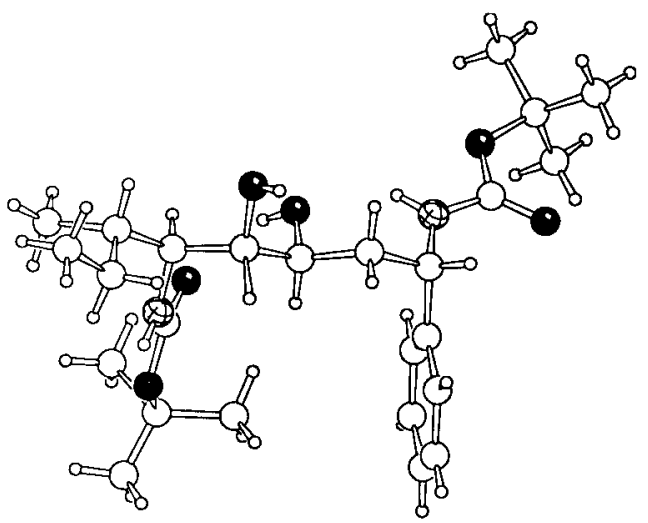

11 (SSSSS)<smiles>CC[C@H](C)[C@H](NC(=O)OC(C)(C)C)[C@@H](O)[C@H](O)C[C@H](NC(=O)OC(C)(C)C)c1ccccc1</smiles>

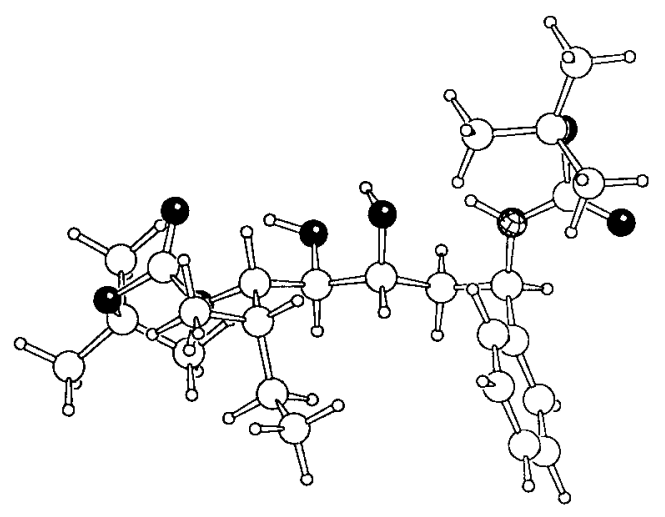

$11(S S R R R)$

Figure 2 The two alternative structures of $\mathbf{1 1}$ obtained on the basis of 2D-NOESY constraints. (oxygens are represented as black, nitrogens as grid)

$\left.\mathrm{CH}_{2}-\mathrm{Ph}\right), 3.75-3.90(\mathrm{~m}, 1 \mathrm{H}, \mathrm{CH}-\mathrm{NH}), 4.30(\mathrm{~d}, 1 \mathrm{H}, \mathrm{J}=10.5$ $\mathrm{Hz}, \mathrm{NH})$, 5.05-5.17 (m, 2H, $\left.\mathrm{CH}_{2}=\right)$, 5.93-6.06 (m, $1 \mathrm{H}$, $\mathrm{CH}=), 7.10-7.70(\mathrm{~m}, 10 \mathrm{H}, \mathrm{Ar}) ;{ }^{13} \mathrm{C} \mathrm{NMR}\left(50.3 \mathrm{MHz}, \mathrm{CDCl}_{3}\right)$ $\delta:-4.79,-4.65,28.11,36.69,57.78,75.72,79.06,111.70$, $125.95,127.76,128.12,129.13,129.40,133.01,134.57$, 139.00, 139.92, 155.98. (4): white solid; m.p. $=65-66^{\circ} \mathrm{C}$. $[\alpha]_{\mathrm{D}}{ }^{20}=-44.5\left(c=2.0, \mathrm{CHCl}_{3}\right) ;{ }^{1} \mathrm{H}$ NMR $\left(300 \mathrm{MHz}, \mathrm{CDCl}_{3}\right)$ $\delta$ : 0.30 (s, 3H, SiMe), 0.33 (s, 3H, SiMe), 0.52 (t, 3H, $J=7.2$ $\mathrm{Hz}, \mathrm{CH}_{3} \mathrm{CH}_{2}$ ), 0.78-0.85 (m, 5H, $\left.\mathrm{CH}_{3} \mathrm{CH}, \mathrm{CH}_{2} \mathrm{CH}_{3}\right), 1.34$ (s, $\left.9 \mathrm{H}, \mathrm{C}\left(\mathrm{CH}_{3}\right)_{3}\right), 1.40-1.70(\mathrm{~m}, 2 \mathrm{H}, \mathrm{CHCH}, \mathrm{OH}), 3.68 .3,78(\mathrm{dd}$, $1 \mathrm{H}, J=10.4,1.9 \mathrm{~Hz}, \mathrm{C} H \mathrm{NH}), 4.50(\mathrm{bd}, 1 \mathrm{H}, J=10.7 \mathrm{~Hz}$ ), 4.78-5.04 (m, 2H, $\left.\mathrm{CH}_{2}=\right)$, 5.88-6.05 (m, $\left.1 \mathrm{H}, \mathrm{CH}=\right)$, 7.25$7.55(\mathrm{~m}, 5 \mathrm{H}, \mathrm{ArH}) ;{ }^{13} \mathrm{C}$ NMR $\left(75.46 \mathrm{MHz}, \mathrm{CDCl}_{3}\right) \delta$ : -4.97, 4.34, 11.40, 17.94, 23.50, 28.34, 36.93, 59.68. 76.39, 78.78, $110.60,127.67,129.28,134.60,136.40,141.25,156.05$.

(9) For discussions of such electronic effects of allylic substituents see: (a) Eyer, M.; Seebach, D. J. Am. Chem. Soc. 1985, 107, 3601. (b) Danishefsky, S.J.; Larson, E.; Springer, J.P. J. Am. Chem. Soc. 1985, 107, 1274.

(10) Curran, D.P.; Gothe, S.A. Tetrahedron 1988, 44, 3945.

(11) Representative procedure: preparation of $(\mathbf{5 a}-\mathbf{b})$ : Triethylamine $(0.27 \mathrm{~mL}, 1.90 \mathrm{mmol})$ and $\mathbf{3}(600 \mathrm{mg}, 1.52$ mmol) were dissolved in $\mathrm{Et}_{2} \mathrm{O}(15 \mathrm{~mL})$. To this solution benzohydroxymoyl chloride $(300 \mathrm{mg}, 1.90 \mathrm{mmol})$ in $\mathrm{Et}_{2} \mathrm{O}(10$ $\mathrm{mL})$ was slowly added $(3.5 \mathrm{~h})$ through a funnel. After the addition was completed the reaction was stirred for $48 \mathrm{~h}$. The solvent was evaporated and the crude was purified by column chromatography on silica gel (petroleum ether : $\mathrm{Et}_{2} \mathrm{O} / 3: 1$ ) affording a $560 \mathrm{mg}(70 \%)$ of two diastereoisomers in the ratio 60:40. Major diastereoisomer $(\mathbf{5 a}): \mathrm{R}_{\mathrm{f}}=0.042 \mathrm{~cm}$ (petroleum ether: $\left.\mathrm{Et}_{2} \mathrm{O}=3: 1\right) .[\alpha]_{\mathrm{D}}{ }^{20}=+23.65\left(c=4.0, \mathrm{CHCl}_{3}\right) ;{ }^{1} \mathrm{H}$ NMR $\left(300 \mathrm{MHz}, \mathrm{CDCl}_{3}\right) \delta: 0.86\left(\mathrm{~s}, 3 \mathrm{H}, \mathrm{Si}-\mathrm{CH}_{3}\right), 0.90(\mathrm{~s}, 3 \mathrm{H}$, $\left.\mathrm{Si}-\mathrm{CH}_{3}\right), 1.50\left(\mathrm{~s}, 9 \mathrm{H}, \mathrm{C}\left(\mathrm{CH}_{3}\right)_{3}\right), 2.96(\mathrm{dd}, 1 \mathrm{H}, J=12.6,10.5$ $\mathrm{Hz}, \mathrm{Ph}-\mathrm{CH}_{2}$ ), 3.16 (s, 1H, OH), 3.32 (dd, $1 \mathrm{H}, J=12.6,3.1 \mathrm{~Hz}$, $\left.\mathrm{Ph}-\mathrm{CH}_{2}\right), 3.42\left(\mathrm{dd}, 1 \mathrm{H}, J=16.8,10.5 \mathrm{~Hz}, \mathrm{CH}_{2}-\mathrm{CH}-\mathrm{O}\right), 3.84$ (dd, $\left.1 \mathrm{H}, J=16.8,9.4 \mathrm{~Hz}, \mathrm{CH}_{2}-\mathrm{CH}-\mathrm{O}\right), 4.10-4.25(\mathrm{~m}, 1 \mathrm{H}, \mathrm{CH}-$ $\mathrm{N}), 4.50(\mathrm{~d}, 1 \mathrm{H}, J=9.4 \mathrm{~Hz}, \mathrm{NH}), 5.34(\mathrm{t}, 1 \mathrm{H}, J=10.5 \mathrm{~Hz}, \mathrm{CH}-$ O), 7.28-8.00 (m, $15 \mathrm{H}, \mathrm{Ar}-\mathrm{H}){ }^{13} \mathrm{C} \mathrm{NMR}\left(50.3 \mathrm{MHz}, \mathrm{CDCl}_{3}\right)$ $\delta:-2.83,-2.63,28.02,35.34,36.83,58.76,73.72,79.46$, $85.47,126.32,126.64,128.03,128.33,128.53,128.83$, $128.38,129.54,129.98,134.77,137.46,138.21,156.35$, 157.62. HRMS (EI): $\mathrm{m} / \mathrm{z}$ found: $517.2529 \mathrm{C}_{30} \mathrm{H}_{27} \mathrm{~N}_{2} \mathrm{O}_{4} \mathrm{Si}$ requires 517.2523. Minor diastereoisomer $(\mathbf{5 b}): \mathrm{R}_{\mathrm{f}}=0.11 \mathrm{~cm}$ (petroleum ether : $\left.\mathrm{Et}_{2} \mathrm{O} / 3: 1\right)$. $[\alpha]_{\mathrm{D}}{ }^{20}=-107.63(c=4.0$, $\mathrm{CHCl}_{3}$ ); ${ }^{1} \mathrm{H} \mathrm{NMR}\left(300 \mathrm{MHz}, \mathrm{CDCl}_{3}\right) \delta: 0.52\left(\mathrm{~s}, 3 \mathrm{H}, \mathrm{Si}-\mathrm{CH}_{3}\right)$, $0.57\left(\mathrm{~s}, 3 \mathrm{H}, \mathrm{Si}-\mathrm{CH}_{3}\right), 1.24\left(\mathrm{~s} 9 \mathrm{H}, \mathrm{C}\left(\mathrm{CH}_{3}\right)_{3}\right), 2.56(\mathrm{dd}, 1 \mathrm{H}$, $J=13.4,8.6 \mathrm{~Hz}, \mathrm{CH}_{2}-\mathrm{Ph}$.), 3.00 (dd, $1 \mathrm{H}, J=16.1,10.1 \mathrm{~Hz}$, $\mathrm{CH}_{2}-\mathrm{CH}-\mathrm{O}$ ), 3.22 (dd, $1 \mathrm{H}, J=13.4,2.2 \mathrm{~Hz}, \mathrm{CH}_{2}-\mathrm{Ph}$ ), 3.30 (bs, $1 \mathrm{H}, \mathrm{OH}), 3.38$ (dd, $1 \mathrm{H}, J=16.1,11.2 \mathrm{~Hz}, \mathrm{CH}_{2}-\mathrm{CH}-\mathrm{O}$ ), $4.26(\mathrm{~d}, 1 \mathrm{H}, J=9.0, \mathrm{NH}), 4.38-4.44(\mathrm{~m}, 1 \mathrm{H}, \mathrm{CH}-\mathrm{N}), 5.40(\mathrm{t}$, $1 \mathrm{H}, J=10.1 \mathrm{~Hz}, \mathrm{CH}-\mathrm{O}), 7.05-7.54(\mathrm{~m}, 15 \mathrm{H}, \mathrm{Ar}-\mathrm{H}) ;{ }^{13} \mathrm{C} \mathrm{NMR}$ $\left(50.3 \mathrm{MHz}, \mathrm{CDCl}_{3}\right) \delta:-3.55,-3.30,28.09,36.33,37.36,57.72$, 73.64, 79.43, 85.02, 126.18, 126.53, 128.07, 128.44, 128.58, $129.07,129.44,129.68,129.99,133.35,134.66,136.81$, 138.37, 156.78, 157.77. $6: \mathrm{R}_{\mathrm{f}}=0.8 \mathrm{~cm}$ (petroleum ether : $\mathrm{Et}_{2} \mathrm{O}$ /3:1); $[\alpha]_{\mathrm{D}}{ }^{20}=-49.30\left(c=3.0, \mathrm{CHCl}_{3}\right) ;{ }^{1} \mathrm{H} \mathrm{NMR}\left(\mathrm{CDCl}_{3}, 200\right.$ $\mathrm{MHz}), \delta: 0.55$ (s, 6H, $\left.\mathrm{SiMe}_{2} \mathrm{Ph}\right), 0.71-0.92\left(\mathrm{~m}, 8 \mathrm{H}, \mathrm{CH}_{3} \mathrm{CH}_{2}\right.$, $\left.\mathrm{CH}_{3} \mathrm{CH}\right), 1.35\left(\mathrm{~s}, 9 \mathrm{H},\left(\mathrm{CH}_{3}\right)_{3} \mathrm{C}\right), 1.55-1.75\left(\mathrm{~m}, 1 \mathrm{H}, \mathrm{CHCH}_{3}\right)$, 2.12 (bs, 1H, OH), 3.16 (dd, $1 \mathrm{H}, J=16.5 \mathrm{~Hz}, 9.5 \mathrm{~Hz}$, $\mathrm{CH}_{2} \mathrm{CHO}$ ), 3.46 (dd, $1 \mathrm{H}, \mathrm{J}=16.5 \mathrm{~Hz}, 10.0 \mathrm{~Hz}, \mathrm{CH}_{2} \mathrm{CHO}$ ), 
$3.82(\mathrm{~m}, 1 \mathrm{H}, \mathrm{CHNH}), 4.76-4.98$ (m, 2H, CH-O+NH), 7.197.39 (m, 6H, ArH), 7.42-7.58 (m, 4H, ArH); ${ }^{13} \mathrm{C} \mathrm{NMR}$ $\left(\mathrm{CDCl}_{3}, 50.3 \mathrm{MHz}\right), \delta:-3.39,-2.61,11.73,18.90,24.70$, 28.26, 36.05, 36.55, 59.77, 74.20, 79.07, 85.69, 126.59, $127.71,127.84,128.47,129.34,134.67,129.95,137.24$, 155.98, 157.50. HRMS (EI): $\mathrm{m} / \mathrm{z}$ found: 496.2748 $\mathrm{C}_{28} \mathrm{H}_{40} \mathrm{~N}_{2} \mathrm{O}_{4} \mathrm{Si}$ requires 496.2757 .

(12) Hudrlik, P.F.; Peterson, D. J. Am. Chem. Soc. 1975, 97, 1464

(13) (a) Kuwajima, I.; Abe, T.; Minami, N. Chem. Lett. 1976, 993. (b) Fleming, I.; Gosh, U. J. Chem. Soc. Perkin Trans. I 1994, 257.

(14) Representative procedure: preparation of 8: To a solution of 5a $(503 \mathrm{mg}, 0.77 \mathrm{mmol})$ in DMF $(20 \mathrm{~mL})$ pyridinium dichromate (PDC) $(1.74 \mathrm{~g}, 4.36 \mathrm{mmol})$ was added under Ar and the mixture was stirred for $18 \mathrm{~h}$. The reaction was quenched with water, the aqueous phase extracted twice with ethyl acetate, and the organic phases were combined, washed twice with water and dried over $\mathrm{MgSO}_{4}$. The drying agent was filtered and the solvent evaporated giving $\mathbf{8}(297 \mathrm{mg}, 98 \%)$ as a yellow oil. From ${ }^{1} \mathrm{H}$ NMR the crude resulted pure enough for being used in the next step without any purification. 8: $[\alpha]_{\mathrm{D}}=+125.8\left(c=3.0, \mathrm{CHCl}_{3}\right) ;{ }^{1} \mathrm{H}$ NMR $\left(200 \mathrm{MHz}, \mathrm{CDCl}_{3}\right)$ $\delta$ : $1.31\left(\mathrm{~s}, 9 \mathrm{H}, \mathrm{C}\left(\mathrm{CH}_{3}\right)_{3}, 2.83\left(\mathrm{dd}, 1 \mathrm{H}, J=13.8,8.9 \mathrm{~Hz}, \mathrm{CH}_{2-}\right.\right.$ $\mathrm{Ph}), 3.21\left(\mathrm{dd}, 1 \mathrm{H}, J=13.8,4.8 \mathrm{~Hz}, \mathrm{CH}_{2}-\mathrm{Ph}\right), 3.47(\mathrm{dd}, 1 \mathrm{H}$, $\left.J=17.2,11.6 \mathrm{~Hz}, \mathrm{CH}_{2}-\mathrm{CH}-\mathrm{O}\right), 3.76(\mathrm{dd}, 1 \mathrm{H}, J=17.2,6.0 \mathrm{~Hz}$, $\mathrm{CH}_{2}$-CH-O), $4.82(\mathrm{dd}, 1 \mathrm{H}, J=13.4,8.3 \mathrm{~Hz}, \mathrm{CH}-\mathrm{NH}), 5.08(\mathrm{~d}$, $1 \mathrm{H}, J=6.5 \mathrm{~Hz}, \mathrm{NH}), 5.32(\mathrm{dd}, 1 \mathrm{H}, J=11.6,6.0 \mathrm{~Hz}, \mathrm{CH}-\mathrm{O})$, 7.15-7.69 (m, $10 \mathrm{H}, \mathrm{Ar}-\mathrm{H}) ;{ }^{13} \mathrm{C}$ NMR $\left(50.3 \mathrm{MHz}, \mathrm{CDCl}_{3}\right) \delta$ : 28.18, 36.28, 36.84, 58.04, 80.22, 83.15, 126.93, 136.35, 155.35, 156.93, 206.39. HRMS (EI): $\mathrm{m} / \mathrm{z}$ found: 394.1894 $\mathrm{C}_{23} \mathrm{H}_{26} \mathrm{~N}_{2} \mathrm{O}_{4}$ requires 394.1892. 9 (98\% yield): $\mathrm{R}_{\mathrm{f}}=0.8$ $\left(\mathrm{CH}_{2} \mathrm{Cl}_{2}: \mathrm{MeOH} / 10: 1\right) ;[\alpha]_{\mathrm{D}}=+77.21\left(c=4.0, \mathrm{CHCl}_{3}\right) ;{ }^{1} \mathrm{H}$ $\mathrm{NMR}\left(\mathrm{CDCl}_{3}, 200 \mathrm{MHz}\right), \delta: 0.69-1.11(\mathrm{~m}, 8 \mathrm{H}$,

$\left.\mathrm{CH}_{3} \mathrm{CH}_{2}+\mathrm{CH}_{3} \mathrm{CH}\right) 1.46\left(\mathrm{~s}, 9 \mathrm{H},\left(\mathrm{CH}_{3}\right)_{3} \mathrm{C}\right), 1.85-2.02(\mathrm{~m}, 1 \mathrm{H}$, $\left.\mathrm{CHCH}_{3}\right), 3.58\left(\mathrm{~m}, 2 \mathrm{H}, \mathrm{CH}_{2}-\mathrm{CH}-\mathrm{O}\right), 4.51(\mathrm{dd}, 1 \mathrm{H}, J=8.7 \mathrm{~Hz}$, $J=5.2 \mathrm{~Hz}, \mathrm{C} H \mathrm{NH}), 5.01(\mathrm{~d}, 1 \mathrm{H}, J=8.7 \mathrm{~Hz}, \mathrm{NH}), 5.12-5.30$ (m, 1H, CH-O), 7.29-7.42 (m, 3H, ArH), 7.58-7.66 (m, 2H, $\mathrm{ArH}) ;{ }^{13} \mathrm{C} \mathrm{NMR}\left(\mathrm{CDCl}_{3}, 50 \mathrm{MHz}\right), \delta: 11.30,16.26,24.03$, 28.17, 35.69, 37.75, 61.98, 79.87, 83.25, 126.6, 128.46, $128.72,130.49,155.70,156.47,207.52$. HRMS (EI): $\mathrm{m} / \mathrm{z}$ found: $360.2052 \mathrm{C}_{20} \mathrm{H}_{28} \mathrm{~N}_{2} \mathrm{O}_{4}$ requires 360.2049 .

(15) Annunziata, R.; Cinquini, M.; Cozzi, F.; Gilardi, A.; Restelli, A. J. Chem. Soc. Perkin Trans. 1 1985, 2289.

(16) Representative procedure: preparation of 10: To a solution of $\mathbf{8}(297 \mathrm{mg}, 0.75 \mathrm{mmol})$ in methanol $(19.3 \mathrm{~mL})$ stirred under $\mathrm{Ar}, \mathrm{NiCl}_{2} \cdot 6 \mathrm{H}_{2} \mathrm{O}(750 \mathrm{mg}, 3.1 \mathrm{mmol})$ was added. The temperature was lowered to $-30{ }^{\circ} \mathrm{C}$ and $\mathrm{NaBH}_{4}(290 \mathrm{mg}, 7.7$ $\mathrm{mmol}$ ) was added in three portions. The reaction mixture was stirred at $-30^{\circ} \mathrm{C}$ for $65 \mathrm{~h}$ in a cryostat, the solvent was evaporated and to the residue $25 \mathrm{~mL}$ of conc. $\mathrm{NH}_{4} \mathrm{OH}$ were added. The aqueous phase was extracted twice with $\mathrm{CH}_{2} \mathrm{Cl}_{2}$ and the combined organic phases were dried over $\mathrm{MgSO}_{4}$, filtered and the solvent evaporated under vacuum. The crude, without any further purification, di-tert-butyl dicarbonate $(188 \mathrm{mg}, 0.86 \mathrm{mmol})$ and triethylamine $(0.13 \mathrm{~mL}, 0.99 \mathrm{mmol})$ were sequentially dissolved in methanol $(16 \mathrm{~mL})$. The reaction mixture was stirred under Ar 4 days at room temperature. The solvent was evaporated and the crude purified by column chromatography over silica gel (petroleum ether: $\mathrm{Et}_{2} \mathrm{O} / 1: 1$ ) giving $134 \mathrm{mg}$ of the largely prevailing diastereoisomer $\mathbf{1 0}$ as a white solid (overall yield for three reaction steps 35\%). 10: $\mathrm{mp} 200-203{ }^{\circ} \mathrm{C} ;[\alpha]_{\mathrm{D}}=-13.3(c=1.5$, $\left.\mathrm{CHCl}_{3}\right) ;{ }^{1} \mathrm{HNMR}\left(200 \mathrm{MHz}, \mathrm{CDCl}_{3}\right) \delta: 1.20\left(\mathrm{~s}, 9 \mathrm{H}, \mathrm{C}\left(\mathrm{CH}_{3}\right)_{3}\right)$,
1.35 (s, 9H, $\left.\mathrm{C}\left(\mathrm{CH}_{3}\right)_{3}\right), 1.70$ (bs, $\left.1 \mathrm{H}, \mathrm{OH}\right), 2.10$ (bs, $\left.1 \mathrm{H}, \mathrm{OH}\right)$, 1.40-1.50 (m, 2H, $\left.\mathrm{CH}_{2} \mathrm{CHPh}\right), 2.40-2.50\left(\mathrm{~m}, 2 \mathrm{H}, \mathrm{PhCH}_{2}\right)$, $3.10\left(\mathrm{~d}, 1 \mathrm{H}, \mathrm{J}=9.9 \mathrm{~Hz}, \mathrm{PhCH}_{2} \mathrm{CHNH}\right), 3.60-3.65(\mathrm{~m}, 1 \mathrm{H}$, $\mathrm{PhCH}_{2} \mathrm{CHCH}$ ), 3.70-3.75 (m, $\left.1 \mathrm{H}, \mathrm{PhCH}_{2} \mathrm{CH}\right), 4.55-4.60$ (m, $\left.1 \mathrm{H}, \mathrm{PhCHCH}_{2} \mathrm{CHOH}\right), 4.90-5.00(\mathrm{~m}, 1 \mathrm{H}, \mathrm{PhCHNH}), 5.10$ (d, $1 \mathrm{H}, \mathrm{J}=10.1 \mathrm{~Hz}, \mathrm{PhCHNH}), 7.00-7.40(\mathrm{~m}, \mathrm{ArH}, 10 \mathrm{H}) ;{ }^{13} \mathrm{C}$ NMR $\left(50.3 \mathrm{MHz}, \mathrm{CDCl}_{3}\right) \delta: 28.33,28.46,36.68,39.08$, $52.14,53.04,67.11,75.26,77.27,79.64,127.73-129.53$, 137.68, 142.06, 156.05, 156.76. HRMS (EI): $\mathrm{m} / \mathrm{z}$ found: 500.2894 $\mathrm{C}_{20} \mathrm{H}_{28} \mathrm{~N}_{2} \mathrm{O}_{4}$ requires 500.2886. 11: White solid: $30 \%$ overall yield for three reaction steps. M.p. $184-186 .{ }^{\circ} \mathrm{C}$; $[\alpha]_{\mathrm{D}}=-11.46\left(c=3.0, \mathrm{CHCl}_{3}\right) ;{ }^{1} \mathrm{H} \mathrm{NMR}\left(200 \mathrm{MHz}, \mathrm{CDCl}_{3}\right)$ $\delta: 0.15-0.20\left(\mathrm{~m}, 1 \mathrm{H}, \mathrm{CH}_{3} \mathrm{CH}_{2}\right), 0.60-0.80\left(2 \mathrm{~S}, 6 \mathrm{H}, \mathrm{CH}_{3}\right.$ $\mathrm{CH}_{2}+\mathrm{CH}_{3} \mathrm{CH}$ ), $1.00-1.10\left(\mathrm{~m}, 1 \mathrm{H}, \mathrm{CH}_{3} \mathrm{CH}_{2}\right), 1.30-1.55$ (m, $\left.19 \mathrm{H}, 2 \mathrm{Me}_{3} \mathrm{C}, \mathrm{CH}_{2} \mathrm{CHPh}\right), 1.85-1.95\left(\mathrm{~m}, 1 \mathrm{H}, \mathrm{CHCH}_{3}\right), 1.95-$ $2.00(\mathrm{~m}, 1 \mathrm{H}, \mathrm{NHCHCHOH}), 2.35-2.40\left(\mathrm{~m}, 1 \mathrm{H}, \mathrm{CH}_{2} \mathrm{CHPh}\right)$, 2.80-2.85 (m, $1 \mathrm{H}, \mathrm{CH}(\mathrm{OH}) \mathrm{CHNH}), 3.40-3.45(\mathrm{~m}, 1 \mathrm{H}$, $\mathrm{CHCHNH}), 3.60-3.65\left(\mathrm{~m}, 1 \mathrm{H}, \mathrm{CH}_{2} \mathrm{CHOH}\right), 4.00-4.10(\mathrm{~d}, 1 \mathrm{H}$, $\mathrm{J}=9.8 \mathrm{~Hz}, \mathrm{CHCHN} H), 4.60\left(\mathrm{~s}, 1 \mathrm{H}, \mathrm{CH}_{2} \mathrm{CHOH}\right), 5.20-5.25$ $(\mathrm{m}, 1 \mathrm{H}, \mathrm{PhCH}), 6.10(\mathrm{~d}, 1 \mathrm{H}, \mathrm{J}=10.0 \mathrm{~Hz}, \mathrm{PhCHNH}), 7.10-$ 7.40 (m, 5H, ArH); ${ }^{13} \mathrm{C}$ NMR $\left(200 \mathrm{MHz}, \mathrm{CDCl}_{3}\right) \delta: 12.05$, 16.65, 22.93, 38.74, 28.10, 28.29, 38.74, 52.37, 57.39, 67.06, 73.30, 79.34, 80.07, 126.09, 128.20, 128.43, 142.25, 155.77, 157.68. HRMS (EI): $\mathrm{m} / \mathrm{z}$ found: $466.3052 \mathrm{C}_{20} \mathrm{H}_{28} \mathrm{~N}_{2} \mathrm{O}_{4}$ requires 466.3043 .

(17) NMR spectra were recorded at $400 \mathrm{MHz}$ in $\mathrm{C}_{6} \mathrm{D}_{6}$. Homonuclear correlations were obtained by the $\mathrm{gCOSY}$ sequence. Heteronuclear correlations were obtained by editedgHSQC sequence. 2D-NOESY spectra were recorded using mixing times of $1.5 \mathrm{~s}$ and $2.0 \mathrm{~s}$. Conformational search was performed with PC-Spartan Pro v1.0.5, using the MMFF Force Field, and then the conformational minima were refined using PC-Model V7.5 and the MMX Force Field.

(18) Crystal Data of 11: $\mathrm{C}_{25} \mathrm{H}_{42} \mathrm{~N}_{2} \mathrm{O}_{6}$ (466.61), Orthorombic, Space group $\mathrm{P} 2{ }_{1} 2_{1} 2_{1}, \mathrm{Z}=4, a=10.8904(6), b=11.2626(6)$, $c=22.5995(12) \AA, V=2771.9(3) \AA^{3}, \mathrm{D}_{\mathrm{c}}=1.118 \mathrm{~g} \mathrm{~cm}^{-3}$, $F(000)=1016, \mu_{\mathrm{Mo}}=0.079 \mathrm{~cm}^{-1}, \mathrm{~T}=298 \mathrm{~K}$. Data were collected using a graphite monochromated Mo-Ka Xradiation $(\lambda=0.71073 \AA)$ in the range $3.25^{\circ}<\theta<30.00^{\circ}$. Of 31346 reflections measured, 8104 were found to be independent $\left(\mathrm{R}_{\text {int }}=0.0847\right), 3670$ of which were considered as observed $\left[\mathrm{F}_{0}>4 \sigma\left(\mathrm{F}_{0}\right)\right]$, and were used in the refinement of 302 parameters leading to a final $R_{1}$ of 0.0546 and a $R_{\text {all }}$ of 0.1141 . The structure was solved by direct method and refined by full-matrix least squares on $F^{2}$, using SHELXTL 97 program packages. In refinements were used weights according to the scheme $\mathrm{w}=\left[\sigma^{2}\left(F_{\mathrm{o}}{ }^{2}\right)+(0.0895 P)^{2}+0.0000 P\right.$ ]$^{-1}$ where $\left.\mathrm{P}=\left(F_{\mathrm{o}}{ }^{2}\right)+2 F_{\mathrm{c}}{ }^{2}\right) / 3$. The hydrogen atoms were located by geometrical calculations and refined using a "riding" method; $w R_{2}$ was equal to 0.1447 . The other atoms were anisotropically refined except the two $t$-butyl groups that were disordered and so splitted into two position and isotropically refined. The goodness of fit parameters $S$ was 0.846 . The largest difference between peak and hole was 0.572 and $-0.272 \mathrm{e}^{-3}$. Crystallographic data (excluding structure factors and including selected torsion angles) have been deposited with the Cambridge Crystallographic Data Center. CCDC-160358.

Article Identifier:

1437-2096,E;2001,0,SI,0995,0998,ftx,en;Y03801ST.pdf 